PROCEEDINGS OF THE

AMERICAN MATHEMATICAL SOCIETY

Volume 131, Number 4, Pages 1043-1048

S 0002-9939(02)06629-7

Article electronically published on July 26, 2002

\title{
ON A THEOREM OF R. STEINBERG ON RINGS OF COINVARIANTS
}

\author{
LARRY SMITH
}

(Communicated by Wolmer V. Vasconcelos)

\begin{abstract}
Let $\rho: G \hookrightarrow \operatorname{GL}(n, \mathbb{F})$ be a representation of a finite group $G$ over the field $\mathbb{F}$. Denote by $\mathbb{F}[V]$ the algebra of polynomial functions on the vector space $V=\mathbb{F}^{n}$. The group $G$ acts on $V$ and hence also on $\mathbb{F}[V]$. The algebra of coinvariants is $\mathbb{F}[V]_{G}=\mathbb{F}[V] / \mathfrak{h}(G)$, where $\mathfrak{h}(G) \subset \mathbb{F}[V]$ is the ideal generated by all the homogeneous $G$-invariant forms of strictly positive degree. If the field $\mathbb{F}$ has characteristic zero, then R. Steinberg has shown (this is the formulation of R. Kane) that $\mathbb{F}[V]_{G}$ is a Poincaré duality algebra if and only if $G$ is a pseudoreflection group. In this note we explore the situation for fields of nonzero characteristic. We prove an analogue of Steinberg's theorem for the case $n=2$ and give a counterexample in the modular case when $n=4$.
\end{abstract}

For a representation $\rho: G \hookrightarrow \operatorname{GL}(n, \mathbb{F})$ of a finite group $G$ over the field $\mathbb{F}$ denote by $\mathbb{F}[V]$ the algebra of polynomial functions on the vector space $V=\mathbb{F}^{n}$. The group $G$ acts on $V$ and hence also on $\mathbb{F}[V]$. The algebra of coinvariants associated to $\rho$ is the algebra $\mathbb{F}[V]_{G}=\mathbb{F}[V] / \mathfrak{h}(G)$, where $\mathfrak{h}(G) \subset \mathbb{F}[V]$ is the Hilbert ideal, i.e., the ideal in $\mathbb{F}[V]$ generated by all the homogeneous $G$-invariant forms of strictly positive degree. (As a general reference for invariant theory we use [10].) In the discussion of coinvariants the following definition has proven useful.

Definition. Let $H$ be a commutative graded connected algebra over a field $\mathbb{F}$. We say that $H$ is a Poincaré duality algebra of formal dimension $d$ if:

- $H_{i}=0$ for $i>d$,

- $\operatorname{dim}_{\mathbb{F}}\left(H_{d}\right)=1$,

- the pairing $H_{i} \otimes H_{d-i} \longrightarrow H_{d}$ given by multiplication is nonsingular, i.e., an element $a \in H_{i}$ is zero if and only if $a \cdot b=0 \in H_{d}$ for all $b \in H_{d-i}$.

If $H$ is a Poincaré duality algebra of dimension $d$, a nonzero element $[H]$ in $H_{d}$ is referred to as a fundamental class for $H$. We write $\mathrm{f}-\operatorname{dim}(H)$ for the formal dimension of $H$.

The notion of Poincaré duality comes from the study of closed manifolds in algebraic topology, and goes back at least to H. Poincaré; see e.g., 6] Section 69. The cohomology of a closed oriented manifold with field coefficients is the prototypical example of a Poincaré duality algebra. In this note we are concerned with the problem of determining under what conditions $\mathbb{F}[V]_{G}$ is a Poincaré duality

Received by the editors April 30, 2001 and, in revised form, November 5, 2001.

1991 Mathematics Subject Classification. Primary 13A50; Secondary 20 F55.

Key words and phrases. Invariant theory, pseudoreflection groups. 
algebra. For further results concerning Poincaré duality quotient algebras of $\mathbb{F}[V]$, with some surprising connections to algebraic topology, see [4].

The portion of Theorem 1.3 of R. Steinberg [11] referred to in the title may be formulated following R. Kane [2] as follows: If the field $\mathbb{F}$ has characteristic zero, then $\mathbb{F}[V]_{G}$ is a Poincaré duality algebra if and only if $G$ is a pseudoreflection group. Pseudoreflection groups have played a central role in invariant theory all along. Since the papers of G. C. Shephard and J. A. Todd [9] and J.-P. Serre [8] it is known that in the nonmodular case these are the groups whose rings of polynomial invariants are again polynomial algebras.

In this note we explore the situation over fields of nonzero characteristic. We prove an analogue of Steinberg's theorem for the case $n=2$, a partial result for $n=3$, and give a counterexample in the modular case when $n=4$.

The essential tools we need for the the degree two case are:

- an elegant application of a theorem of J.-P. Serre [7] by W. Vasconcelos [12],

- an ancient argument due to D. Hilbert [1,

- and a very special property of rings of invariants in the degree two case which seems not to have been observed before.

The result of W. Vasconcelos, 12, Proposition 2.4, in our case runs as follows.

Proposition 1 (W. Vasconcelos). Let $\mathbb{F}$ be a field and $I$ an $\overline{\mathbb{F}[x, y]}$-primary irreducible ideal. Then $I$ is generated by two elements which form a regular sequence in $\mathbb{F}[x, y]$.

The relevance of this result for our problem is the following observation.

Lemma 2. Let $H$ be a Poincaré duality algebra over the field $\mathbb{F}$. Assume that $H \cong \mathbb{F}[V] / I$ for some finite dimensional vector space $V$ over $\mathbb{F}$. Then $I \subset \mathbb{F}[V]$ is an irreducible $\overline{\mathbb{F}[V]}$-primary ideal.

Proof. Let $[H] \in H_{d}$ be a fundamental class. The vector space spanned by $[H]$ is the unique nonzero minimal ideal of $H$. This means that $I$ has a unique minimal proper over ideal and therefore $I$ is irreducible (see e.g. [13] Chapter IV Section $16)$.

We next record a very special property of rings of invariants in degree two.

Proposition 3. Let $\rho: G \hookrightarrow \mathrm{GL}(2, \mathbb{F})$ be a representation of a finite group over the field $\mathbb{F}$. Then the inclusion $i: \mathbb{F}[x, y]^{G} \hookrightarrow \mathbb{F}[x, y]$ is split by an $\mathbb{F}[x, y]^{G}$-module homomorphism.

Proof. If $\mathbb{F}$ has characteristic prime to the order of the group, the splitting may be obtained from the transfer homomorphism $\operatorname{Tr}^{G}: \mathbb{F}[x, y] \longrightarrow \mathbb{F}[x, y]^{G}$ by dividing by the order of the group [10], Section 2.4. Otherwise, let $P=\operatorname{Syl}_{p}(G)$ be a $p$-Sylow subgroup of $G$. Up to conjugation $\rho(P)$ is a finite subgroup of

$$
\operatorname{Uni}(2, \mathbb{F})=\left\{\left[\begin{array}{ll}
1 & \lambda \\
0 & 1
\end{array}\right] \mid \lambda \in \mathbb{R}\right\}
$$

so $\rho(P)$ is a Landweber-Stong group; see $\left[10\right.$, Section 8.2. Hence $\mathbb{F}[x, y]^{P}$ is a polynomial algebra and $\mathbb{F}[x, y]$ is a free $\mathbb{F}[x, y]^{P}$-module, loc.cit. Therefore there exists an $\mathbb{F}[x, y]^{P}$-module splitting

$$
\pi: \mathbb{F}[x, y] \longrightarrow \mathbb{F}[x, y]^{P}
$$


to the inclusion $\mathbb{F}[x, y]^{P} \hookrightarrow \mathbb{F}[x, y]$. The composition with the relative transfer $\operatorname{Tr}_{P}^{G}$, viz.,

$$
\mathbb{F}[x, y] \stackrel{\pi}{\longrightarrow} \mathbb{F}[x, y]^{P} \stackrel{\operatorname{Tr}_{P}^{G}}{\longrightarrow} \mathbb{F}[x, y]^{G}
$$

when restricted to $\mathbb{F}[x, y]^{G}$ is multiplication by the index $|G: P|$ of $P=\operatorname{Syl}_{P}(G)$ in $G$ which is prime to $p$, so dividing by $|G: P|$ gives the desired splitting.

The next result is the analog of R. Steinberg's theorem in the degree two case.

Theorem 4. Let $\rho: G \hookrightarrow \mathrm{GL}(2, \mathbb{F})$ be a representation of a finite group $G$ over the field $\mathbb{F}$. If $\mathbb{F}[x, y]_{G}$ is a Poincaré duality algebra, then it is a complete intersection and the Hilbert ideal $\mathfrak{h}(G)$ is generated by two invariant forms $f, h \in \mathbb{F}[x, y]^{G}$ which form a regular sequence in $\mathbb{F}[x, y]$, and $\mathbb{F}[x, y]^{G}=\mathbb{F}[f, h]$ is a polynomial algebra so $G$ is generated by pseudoreflections.

Proof. If $\mathbb{F}[x, y]_{G}$ is a Poincaré duality algebra, then the Hilbert ideal $\mathfrak{h}(G) \subset$ $\mathbb{F}[x, y]$ is an irreducible $\overline{\mathbb{F}[x, y]}$-primary ideal so by W. Vasconcelos' Proposition [12], Proposition 2.4 (quoted here as Proposition 1), the Hilbert ideal $\mathfrak{h}(G)$ is generated by two forms $f, h$ that form a regular sequence in $\mathbb{F}[x, y]$. If we suppose that $\operatorname{deg}(f) \leq \operatorname{deg}(h)$, then for degree reasons we have $f \in \mathbb{F}[x, y]^{G}$. We claim that it is possible to choose $h \in \mathbb{F}[x, y]^{G}$ also. Granted this we may complete the proof by using an argument of D. Hilbert [1] as follows: By Proposition 2 there is an $\mathbb{F}[x, y]^{G}$-module homomorphism $\sigma: \mathbb{F}[x, y] \longrightarrow \mathbb{F}[x, y]^{G}$ splitting the inclusion $\mathbb{F}[x, y]^{G} \subseteq \mathbb{F}[x, y]$. Let $d \in \mathbb{N}$ be the smallest integer for which there is an invariant form of strictly positive degree. For degree reasons $\mathbb{F}[x, y]^{G}$ and $\mathbb{F}[f, h]$ agree in degree $d$. Suppose that we have shown that $\mathbb{F}[x, y]_{k}^{G}=\mathbb{F}[f, h]_{k}$ for $d \leq k<\ell$ and let $F \in \mathbb{F}[x, y]_{\ell}^{G}$ be an invariant form of degree $\ell$. Since $F \in \mathfrak{h}(G)$ we may write $F=\Phi f+\Psi h$ with $\Phi, \Psi \in \mathbb{F}[x, y]$ forms of positive degree. If we apply $\sigma$ to this equation, then, since $F, f$, and $h$ are $G$-invariant we get $F=\sigma(\Phi) f+\sigma(\Psi) h$. The forms $\sigma(\Phi)$ and $\sigma(\Psi)$ are $G$-invariant and have degree strictly less than $\ell$, so by the inductive hypothesis belong to $\mathbb{F}[f, h]$. Hence so does $F$. Thus $\mathbb{F}[x, y]^{G}=\mathbb{F}[f, h]$ is a polynomial algebra and therefore $\rho(G)$ is generated by pseudoreflections by a theorem of J.-P. Serre [8].

So it remains to show that we may choose $h$ to be a $G$-invariant form. Choose a minimal set of algebra generators $f=\phi_{1}, \phi_{2}, \ldots, \phi_{m} \in \mathbb{F}[x, y]^{G}$ for $\mathbb{F}[x, y]^{G}$, with $\operatorname{deg}\left(\phi_{1}\right) \leq \operatorname{deg}\left(\phi_{2}\right) \leq \cdots \leq \operatorname{deg}\left(\phi_{m}\right)$. Suppose that $\operatorname{deg}\left(\phi_{2}\right)<\operatorname{deg}(h)$. Then, since $\phi_{2} \in \mathfrak{h}(G)=(f, h)$ we must have for degree reasons that $\phi_{2}=f_{2} \cdot f$ for some $f_{2} \in \mathbb{F}[x, y]$. Since $\phi_{2}$ and $f$ are invariant so is $f_{2}$, but this contradicts the indecomposability of $\phi_{2}$. Therefore $\operatorname{deg}(h) \leq \operatorname{deg}\left(\phi_{2}\right) \leq \cdots \leq \operatorname{deg}\left(\phi_{m}\right)$ and, since $h \in \mathfrak{h}(G)$, we may write

$$
h=h_{1} \phi_{1}+h_{2} \phi_{2}+\cdots+h_{k} \phi_{k}
$$

where $\operatorname{deg}\left(\phi_{2}\right)=\cdots=\operatorname{deg}\left(\phi_{k}\right)=\operatorname{deg}(h)$. If we set

$$
\phi=h-h_{1} \phi_{1}=h_{2} \phi_{2}+\cdots+h_{k} \phi_{k},
$$

then $\phi \equiv h \bmod (f)$ so $\mathfrak{h}(G)=(f, \phi), f, \phi \in \mathbb{F}[x, y]$ a regular sequence. Moreover $h_{2}, \ldots, h_{k}$ are scalars since they are of degree zero, and therefore $\phi$ is an $\mathbb{F}$-linear combination of the invariant forms $\phi_{2}, \ldots, \phi_{k}$ so $\phi \in \mathbb{F}[x, y]^{G}$, which shows that $h$ may be chosen to be a $G$-invariant form, and completes the proof. 
If $P \hookrightarrow \operatorname{GL}(n, \mathbb{F})$ is a representation of a finite $p$-group over the field $\mathbb{F}$ of characteristic $p$, then the action of $P$ on the space of linear forms always has a nonzero fixed point. If $0 \neq z \in\left(V^{*}\right)^{P}$ is such a fixed point, then

$$
\mathbb{F}[V]_{P}=\frac{\mathbb{F}[V]}{\mathfrak{h}(P)} \cong \frac{\mathbb{F}[V] /(z)}{\mathfrak{h}(P) /(z)} .
$$

So, for $n=3$ the coinvariants $\mathbb{F}[x, y, z]_{P}$ are actually a quotient of $\mathbb{F}[x, y]$. Hence Vasconcelos' proposition [12], Proposition 2.4 (quoted here as Proposition 1), shows that if $\mathbb{F}[x, y, z]_{P}$ is a Poincaré duality algebra, then $\mathfrak{h}(P)$ is generated by three elements that form a regular sequence in $\mathbb{F}[x, y, z]$ and $\mathbb{F}[x, y, z]_{P}$ is a complete intersection. Hence we have shown

Corollary 5. If $P \hookrightarrow \mathrm{GL}(3, \mathbb{F})$ is a representation of a finite p-group over the field $\mathbb{F}$ of characteristic $p$ and $\mathbb{F}[x, y, z]_{P}$ is a Poincaré duality algebra, then $\mathfrak{h}(P)$ is generated by three elements that form a regular sequence in $\mathbb{F}[x, y, z]$.

Therefore for $p$-groups the first degree in which Steinberg's Theorem can fail, even in the weak sense that the Hilbert ideal fails to be generated by a regular sequence, is degree four, and in degree four Steinberg's Theorem fails in this weak sense for the vector invariants of $\mathbb{Z} / 2$ over $\mathbb{F}_{2}$. This much-used example has a number of other interesting properties in this connection that we then briefly indicate.

Example. Consider the permutation representation of $\mathbb{Z} / 2$ on the set $X_{Y}(2)=$ $\left\{x_{1}, y_{1}, x_{2}, y_{2}\right\}$ where the nonidentity element of $\mathbb{Z} / 2$ acts on $X_{Y}(2)$ by simultaneous interchange of $x_{1}, x_{2}$ with $y_{1}, y_{2}$, i.e., if $\tau \in \mathbb{Z} / 2$ is the nonidentity element, then

$$
\begin{aligned}
& \tau\left(x_{i}\right)=y_{i} \text { for } i=1,2, \text { and } \\
& \tau\left(y_{j}\right)=x_{j} \text { for } j=1,2 .
\end{aligned}
$$

We consider this permutation representation as defining a linear representation of $\mathbb{Z} / 2 \hookrightarrow \mathrm{GL}\left(4, \mathbb{F}_{2}\right)$ of degree 4 over the Galois field $\mathbb{F}_{2}$ with 2 elements. The ring of invariants $\mathbb{F}_{2}\left[X_{Y}(2)\right]^{\mathbb{Z} / 2}$ is known (see e.g., [10], Chapter IV, Section 3, Example 4); it is generated by five polynomials

$$
x_{1}+y_{1}, x_{1} y_{1}, x_{2}+y_{2} x_{2} y_{2}, x_{1} y_{2}+x_{2} y_{1} .
$$

The formulas

$$
\begin{aligned}
& x_{2}\left(x_{1}+y_{1}\right)=x_{2} x_{1}+x_{2} y_{1}, \\
& x_{1}\left(x_{2}+y_{2}\right)=x_{1} x_{2}+x_{1} y_{2},
\end{aligned}
$$

when added together show that $x_{1} y_{2}+x_{2} y_{1}$ belongs to the ideal in $\mathbb{F}_{2}\left[x_{1}, y_{1}, x_{2}, y_{2}\right]$ generated by the four polynomials $x_{1}+y_{1}, x_{1} y_{1}, x_{2}+y_{2}, x_{2} y_{2}$, so these four polynomials, which are a regular sequence in $\mathbb{F}_{2}\left[x_{1}, y_{1}, x_{2}, y_{2}\right]$, generate the Hilbert ideal $\mathfrak{h}(\mathbb{Z} / 2)$. Hence $\mathbb{F}_{2}\left[x_{1}, y_{1}, x_{2}, y_{2}\right]_{\mathbb{Z} / 2}$ is a Poincaré duality algebra, although the ring of invariants $\mathbb{F}_{2}\left[x_{1}, y_{1}, x_{2}, y_{2}\right]^{\mathbb{Z} / 2}$ is not a polynomial algebra, and $\mathbb{Z} / 2$ contains no pseudoreflections.

This example has a number of other interesting properties.

Although the four polynomials $x_{1}+y_{1}, x_{1} y_{1}, x_{2}+y_{2}, x_{2} y_{2}$ generate the Hilbert ideal $\mathfrak{h}(\mathbb{Z} / 2)$ they do not generate the ring of invariants $\mathbb{F}_{2}\left[x_{1}, y_{1}, x_{2}, y_{2}\right]^{\mathbb{Z} / 2}$. Furthermore, if we denote by $\rho: \mathbb{Z} / 2 \hookrightarrow \mathrm{GL}\left(2, \mathbb{F}_{2}\right)$ the linearization of the permutation representation of $\mathbb{Z} / 2$ on $X_{\mathrm{Y}}=\{x, y\}$ where $\tau \in \mathbb{Z} / 2$ interchanges $x$ and $y$, then $\rho \times \rho: \mathbb{Z} / 2 \times \mathbb{Z} / 2 \hookrightarrow \mathrm{GL}\left(4, \mathbb{F}_{2}\right)$ is a faithful representation of $\mathbb{Z} / 2 \times \mathbb{Z} / 2$ of degree 4 . 
Choose a basis $x_{1}, y_{1}, x_{2}, y_{2}$ for $\mathbb{F}_{2}{ }^{4}$ so that the generators $\tau \times 1$ and $1 \times \tau$ act by the rules:

$$
\begin{aligned}
& (\tau \times 1)\left(x_{1}\right)=y_{1} \\
& (\tau \times 1)\left(y_{1}\right)=x_{1} \\
& (\tau \times 1)\left(x_{2}\right)=x_{2} \\
& (\tau \times 1)\left(y_{2}\right)=y_{2} \\
& (1 \times \tau)\left(x_{1}\right)=x_{1} \\
& (1 \times \tau)\left(y_{1}\right)=y_{1} \\
& (1 \times \tau)\left(x_{2}\right)=y_{2} \\
& (1 \times \tau)\left(y_{2}\right)=x_{2} \text {. }
\end{aligned}
$$

For the ring of invariants one obtains that

$$
\mathbb{F}_{2}\left[x_{1}, y_{1}, x_{2}, y_{2}\right]^{\mathbb{Z} / 2 \times \mathbb{Z} / 2}=\mathbb{F}_{2}\left[x_{1}+y_{1}, x_{1} y_{1}, x_{2}+y_{2}, x_{2} y_{2}\right]
$$

and so

$$
\mathfrak{h}(\mathbb{Z} / 2 \times \mathbb{Z} / 2)=\left(x_{1}+y_{1}, x_{1} y_{1}, x_{2}+y_{2}, x_{2} y_{2}\right)=\mathfrak{h}(\mathbb{Z} / 2) .
$$

Passing to the quotients by the Hilbert ideal gives for the coinvariant algebras

$$
\mathbb{F}_{2}\left[x_{1}, y_{1}, x_{2}, y_{2}\right]_{\mathbb{Z} / 2 \times \mathbb{Z} / 2} \cong \mathbb{F}_{2}\left[x_{1}, y_{1}, x_{2}, y_{2}\right]_{\mathbb{Z} / 2} .
$$

This means the two distinct groups, $\mathbb{Z} / 2$ and $\mathbb{Z} / 2 \times \mathbb{Z} / 2$, of distinct orders, each have a four dimensional representation for which the Hilbert ideals coincide, and hence for which their rings of coinvariants are isomorphic. The coinvariants are a complete intersection; one group is generated by reflections and has polynomial invariants, the other contains no reflections and its ring of invariants is not a polynomial algebra.

In closing we note that in the nonmodular case it has been shown by T.-C. Lin [3] that the ring of coinvariants of a representation $\rho: G \hookrightarrow \operatorname{GL}(n, \mathbb{F})$ is a Poincaré duality algebra if and only if the ring of invariants is a polynomial algebra. From the theorem of Shephard and Todd (see, e.g. [10, Theorem 7.4.1) this in turn is equivalent to $\rho(G)$ being generated by pseudoreflections. Taken together with the results demonstrated this here raises the problem of characterizing those representations $\rho: G \hookrightarrow \operatorname{GL}(n, \mathbb{F})$ for which $\mathbb{F}[V]_{G}$ is a Poincaré duality algebra. In particular what happens in the case of degree three for groups of composite order?

\section{REFERENCES}

1. D. Hilbert, "Uber die Theorie der Algebraischen Formen, Math. Annalen 36 (1890), 473-534.

2. R. Kane, Poincaré Duality and the Ring of Coinvariants, Canad. Math. Bull. 37 (1994), 82-88. MR 96e:51016

3. T.-C. Lin, Poincarédualität und Ringen von Koinvarianten, Universität Göttingen, Doktorarbeit, (to appear).

4. D. M. Meyer and L. Smith, Poincaré Duality Algebras, Macaulay's Dual Systems and Steenrod Operations, AG-Invariantentheorie, (to appear).

5. M. D. Neusel and L. Smith, Invariant Theory of Finite Groups, Mathematical Surveys and Monographs, American Mathematical Society, Providence, RI, 2002. CMP 2002:05

6. H. Seifert and W. Threlfall, Lehrbuch der Topologie, Chelsea Publ. Co., New York, NY, 1947.

7. J. P. Serre, Sur les Modules Projectifs, Sem. P. Dubriel, M.L. Dubriel-Jacotin et C. Pisot, 14ème anné (1960/61), ENS Paris, 1961.

8. J. -P. Serre, Groupes finis d'automorphismes d'anneaux locaux réguliers, Colloq. d'Alg. Éc. Norm. Sup. de Jeunes Filles, Paris, 8-01-8-11, 1967. MR 38:3267]

9. G. C. Shephard and J. A. Todd, Finite Unitary Reflection Groups, Can. J. of Math. 6 (1954), 274-304. MR 15:600b

10. L. Smith, Polynomial Invariants of Finite Groups, A.K. Peters, Ltd., Wellesley, MA, 1995, second printing 1997. MR 96f:13008

11. R. Steinberg, Differential Equations Invariant under Finite Reflection Groups, Trans. of the American Mathematical Society 112 (1964), 392-400. MR 29:4807 
12. W. V. Vasconcelos, Ideals Generated by R-Sequences, J. of Algebra 6 (1967), 309-316. MR 35:4209

13. O. Zariski and P. Samuel, Commutative Algebra, Volumes I,II, Graduate Texts in Math. 28, 29, Springer-Verlag, Berlin, New York, 1975. MR 52:5641 MR 52:10706

AG-Invariantentheorie, Mathematisches Institut der Universität, Bunsenstrasse 35, D37073 Göttingen, Federal Republic of Germany

E-mail address: larry@sunrise.uni-math.gwdg.de 